\title{
Ultrasonic evaluation of renal cortex arterial area enables differentiation between hypertensive and glomerulonephritis-related chronic kidney disease
}

\author{
Arkadiusz Lubas ${ }^{1}$ - Grzegorz Kade ${ }^{1} \cdot$ Robert Ryczek $^{2} \cdot$ Piotr Banasiak $^{3}$. \\ Przemysław Dyrla $^{4} \cdot$ Katarzyna Szamotulska $^{5} \cdot$ Daniel Schneditz $^{6}$. \\ Stanisław Niemczyk ${ }^{1}$
}

Received: 10 March 2017 / Accepted: 27 May 2017 / Published online: 1 June 2017

(c) The Author(s) 2017. This article is an open access publication

\begin{abstract}
Purpose Identifying the primary etiology of cardio-renal syndrome in a timely manner remains an ongoing challenge in nephrology. We hypothesized that hypertensive kidney damage can be distinguished from chronic glomerulonephritis at an early stage of chronic kidney disease (CKD) using ultrasound (US) Doppler sonography.

Methods Fifty-six males (age $54 \pm 15$, BMI $28.3 \pm 3.5 \mathrm{~kg}$ / $\mathrm{m}^{2}$ ) with hypertension and stable CKD at stages 2-4 [38 with essential hypertension (HT-CKD); 18 with glomerulonephritis (GN-CKD)] were studied. Blood tests, UACR, echocardiography, ABPM, carotid IMT, and an ultrasound dynamic tissue perfusion measurement (DTPM) of the renal cortex were performed.

Results HT-CKD patients had reduced proximal renal cortex perfusion as well as reduced total and proximal renal cortex arterial area. Proximal renal cortex arterial area $\leq 0.149 \mathrm{~cm}^{2}$ identified hypertension-related CKD with a
\end{abstract}

Arkadiusz Lubas

alubas@wim.mil.pl

1 Department of Internal Diseases, Nephrology and Dialysis, Military Institute of Medicine, Szaserów 128, 04-141 Warsaw 44, Poland

2 Department of Cardiology, Military Institute of Medicine, Szaserów 128, 04-141 Warsaw 44, Poland

3 Health Center Karczew, Otwocka 28, 05-480 Karczew, Poland

4 Department of Gastroenterology, Military Institute of Medicine, Szaserów 128, 04-141 Warsaw 44, Poland

5 Department of Epidemiology, Institute of Mother and Child, Kasprzaka 17a, 01-211 Warsaw, Poland

6 Institute of Physiology, Medical University of Graz, Harrachgasse 21/5, 8010 Graz, Austria sensitivity of $71 \%$ and a specificity of $78 \%$ (AUC 0.753 , $p<0.001$ ).

Conclusions Evidence of diminished arterial vascularity or perfusion of renal proximal cortex, both derived from US Doppler, could be helpful in differentiating hypertensive nephropathy from glomerulonephritis-related CKD.

Keywords Renal perfusion · Hypertension · Arterial rarefaction · Ultrasound Doppler

\section{Introduction}

Changes in renal perfusion play an important part in the development of cardio-renal syndrome [1, 2]. Both longstanding hypertension and a history of malignant hypertension are well known to induce chronic kidney damage. On the other hand, kidney disease may cause or aggravate an existing hypertension. Vascular changes in the course of hypertension are significant diagnostically and constitute important additional cardiovascular risk factors [3]. In large arteries such changes include thickening of the whole vessel wall and its intima-media layers. In small arteries changes are characterized by wall hypertrophy and vascular rarefaction [4]. It also has been shown that cardiac dynamics have a significant influence on renal perfusion and function [5, 6]. On the other hand, etiology of renal impairment and related secondary hypertension due to chronic, nonvascular-mediated glomerulonephritis is different.

Within the last years there has been significant progress in the field of ultrasound (US) imaging and the widespread availability of advanced US equipment has considerably extended the potential of US Doppler diagnostics. It has been recently shown that dynamic Doppler perfusion measurements (DTPM) can be used to differentiate between 
inflammatory and proliferative changes in the pancreas [7]. US-derived markers of renal perfusion disorders have been used to diagnose focal lesions such as abscesses, cysts, and tumors, as well as lesions affecting the whole kidney such as ischemic and diabetic nephropathy, transplant rejection, and obstructive uropathy, or cardiac disorders such as hypertensive organ damage or systolic heart failure [8-11].

We therefore hypothesized that vascular-induced hypertensive kidney damage leads to perfusion changes that can be distinguished from those of chronic glomerulonephritis. The aim of the study was to evaluate the differences in US Doppler parameters of renal cortex perfusion in hypertensive and glomerulonephritis-related kidney damage.

\section{Materials and methods}

Patients with hypertension and stable CKD during last 3 months, at stages 2-4 were recruited from consecutive subjects admitted to the Department of Internal Diseases Nephrology and Dialysis. Exclusion criteria were defined as (a) cause of kidney damage other than glomerulonephritis or hypertension, or (b) the presence of acute cardiac and renal diseases, or (c) history of renal artery stenosis, CKD stage 5, heart failure in NYHA III or IV stage, atrial fibrillation, hyperkinetic state, inflammation, connective tissue diseases, vasculitis, diabetes mellitus, amyloidosis, hydronephrosis, systemic cancer, or (d) inability to obtain good-quality US images of renal or cardiac structures. Stages of CKD were defined as per K/DOQI guidelines [12]. On the basis of anamnesis, previously and actually performed blood and urinary tests patients were classified into hypertension-related kidney damage group (HT-CKD) if they had long-standing or history of severe hypertension preceding the renal impairment without nephrotic proteinuria. Patients with previously recognized glomerulonephritis (performed renal biopsy or CKD with persistent microhematuria and/or proteinuria and excluded other causes of kidney damage) were classified into GN-CKD group.

The local bioethics committee approved the protocol of the study (35/WIM/2011, June 15, 2016). All participants enrolled in the study provided written informed consent.

\section{Blood and urinary tests}

Serum creatinine (Cr) and cystatin C (Cys) were measured, and glomerular filtration rate was estimated (eGFR) using the chronic kidney disease epidemiology (CKDEPI) formula [13]. The presence of inflammation and cardiac function was assessed by measuring C-reactive protein (CRP), Troponin-I, and N-terminal prohormone of brain natriuretic peptide (NT-proBNP) levels. Urinary albumin-to-creatinine ratio $(\mathrm{mg} / \mathrm{dL} / \mathrm{mg} / \mathrm{dL})$ (UACR) from first-morning urine sample was calculated.

\section{Blood pressure monitoring}

Systolic and diastolic blood pressures were measured every $15 \mathrm{~min}$ at daytime and every $30 \mathrm{~min}$ at night using the ambulatory blood pressure monitor (ABPM-04, Meditech, Hungary) and averaged over the whole daily cycle.

\section{Kidney ultrasound}

A Logiq P6 (GE Healthcare, Korea) US device and a convex transducer $4 \mathrm{~L}(2-5 \mathrm{MHz})$ were used for a renal examination. Maximal right renal length and mean cortical thickness ( 2 measurements in upper and lower pole) in longitudinal axis were examined. Renal resistance indices (RI) in segmental arteries of both kidneys were calculated from two to three flow measurements in the upper, middle, and lower regions of the renal sinus. When the clinical data suggested renal artery stenosis, a full Doppler examination of renal and intrarenal arteries was performed.

Color Doppler renal cortical perfusion assessments were made as described elsewhere [5, 6]. Longitudinal sections of right kidneys were examined because of improved access to the mid-segment of the renal cortex. Left kidneys were not examined to avoid possible artifacts caused by preceding renal biopsies. Renal perfusion was estimated using the DTPM method [14]. Renal arterial perfusion $(\mathrm{mL} / \mathrm{s})$ and renal arterial area $\left(\mathrm{cm}^{2}\right)$ obtained from the best quality color Doppler video clip, selected from 2 to 3 recorded DICOM sequences, covering a minimum of three heart cycles in the chosen region of interest (ROI) were calculated using the PixelFlux (Chameleon-Software, Leipzig, Germany) software package. Color Doppler frequency was constantly set at $3.4 \mathrm{MHz}$. The ROI was set in the midsegment of the renal cortex between the outer border of medullary pyramids and the kidney surface avoiding focal abnormalities. The ROI sample contained vessels pointing to the transducer so that angle correction was not required. Total renal cortex perfusion (RCP) and total renal cortex arterial area (RCAA) were calculated from the whole ROI. Then the ROI was divided into equally sized proximal and distal regions. Regional perfusions and arterial areas were separately measured and then considered for statistics. To avoid errors resulting from different ROI sizes, proximal renal cortex arterial area was normalized to proximal ROI creating an arterial area index (AAI). This index is thought as a measure of arterial vascularity, a substitute of 3D arterial density in 2D US. 


\section{Carotid sonography}

To determine the degree of hypertensive organ damage left common carotid artery intima-media thickness (cIMT) was measured manually at least $10 \mathrm{~mm}$ upstream of the carotid sinus using a $11 \mathrm{~L}$ transducer $(10-13 \mathrm{MHz})$ [5]. The mean of three measurements was calculated.

\section{Cardiac sonography}

For echocardiography the Vivid S6 system (GE Healthcare, USA) was used with a M4S-RS transducer (1.5-3.6 MHz). All M-mode measurements were taken according to recommendations of the American Society of Echocardiography [15]. The left atrial (LA) diameter was measured using M-mode methodology from the parasternal long axis view. Left ventricular mass (LVM) was calculated from the Devereux et al. formula and then normalized (LVMI) for Mosteller's body surface area [16, 17]. Left ventricular ejection fraction (LVEF) was estimated by Simpson's biplane method [18]. Left ventricular cardiac index (CI) was collected using 2D/continuous US Doppler functions. Peak values of early (E) and late (A) parts of mitral inflow velocities were measured. Tissue Doppler early diastolic mitral annular velocity $\left(\mathrm{E}^{\prime}\right)$ was measured from the apical four-chamber view with a 4-mm sample volume placed at the septal corner of mitral annulus. Then $\mathrm{E} / \mathrm{A}$ and $\mathrm{E} / \mathrm{E}^{\prime}$ ratios were calculated.

\section{Statistical analysis}

Variables were analyzed with Pearson's or Spearman's correlation test depending on the type of distribution. Accordingly, the parametric $T$ test or the nonparametric $\mathrm{U}$
Mann-Whitney test was used to analyze the difference of continuous variables between groups. The Chi-square test was performed to determine differences in hypertensive treatment. Stepwise multivariable linear regression analyses were used to identify the factors independently associated with proximal RCP and proximal RCAA. Receiver operating characteristic (ROC) analyses were performed to identify thresholds of proximal RCP and RCAA to differentiate between hypertensive and glomerulonephritisrelated chronic kidney disease. Statistica 12 (StatSoft Inc., Poland) software was used for statistical analysis.

\section{Results}

Sixty males with hypertension and stable CKD ranging from stages 2 to 4 entered the study. Four patients were excluded, three because of unexpected diabetes mellitus and one patient because of failure to obtain echocardiographic Doppler data, so that 56 patients (age $54 \pm 15$, BMI $28.3 \pm 3.5 \mathrm{~kg} / \mathrm{m}^{2}$ ) entered final analysis. Chronic kidney disease caused by essential hypertension (HT-CKD) was found in 38 patients, whereas chronic kidney disease originating from glomerulonephritis (GN-CKD) was found in 18 patients. 9 HT-CKD and 2 GN-CKD patients underwent full Doppler examinations of renal arteries with the exclusion of renal artery narrowing. In all patients the difference between right and left renal RI did not exceed 0,05 [19].

HT-CKD patients were older and had lower UACR, but the kidney length, cortical thickness, and function were similar in both groups (Table 1). Groups did not differ in antihypertensive or nephroprotective treatment (Table 2).

Table 1 Patient and renal characteristics

\begin{tabular}{|c|c|c|c|c|c|}
\hline Data & Total $(n=56)$ & $\operatorname{HT}-\mathrm{CKD}(n=38)$ & $\begin{array}{l}\text { HT-CKD matched } \\
(n=18)\end{array}$ & GN-CKD $(n=18)$ & $\begin{array}{l}p \text { value } \mathrm{HT} / \mathrm{GN} \\
(\mathrm{HTm} / \mathrm{GN})\end{array}$ \\
\hline Age (y) & $53.96 \pm 14.89$ & $58.08 \pm 13.37$ & $47.4 \pm 12.12$ & $45.3 \pm 14.11$ & $0.003(0.728)$ \\
\hline BMI $\left(\mathrm{kg} / \mathrm{m}^{2}\right)$ & $28.31 \pm 3.46$ & $28.83 \pm 3.41$ & $29.28 \pm 3.95$ & $27.26 \pm 3.32$ & $0.106(0.114)$ \\
\hline Cystatin (mg/L) & $1.54 \pm 0.72$ & $1.42 \pm 0.66$ & $1.43 \pm 0.62$ & $1.73 \pm 0.83$ & $0.187(0.351)$ \\
\hline Creatinine $(\mathrm{mg} / \mathrm{dL})$ & $1.83 \pm 0.78$ & $1.68 \pm 0.73$ & $1.78 \pm 0.91$ & $2.08 \pm 0.81$ & $0.054(0.217)$ \\
\hline $\begin{array}{l}\text { CKD-EPICys-Cr (mL/ } \\
\left.\mathrm{min} / 1.73 \mathrm{~m}^{2}\right)\end{array}$ & $54.29 \pm 27.91$ & $58.55 \pm 28.79$ & $63.00 \pm 34.43$ & $48.50 \pm 25.90$ & $0.210(0.210)$ \\
\hline $\begin{array}{l}\text { Urea/creatinine }(\mathrm{mg} / \mathrm{dL} / \\
\mathrm{mg} / \mathrm{dL})\end{array}$ & $36.36 \pm 9.26$ & $37.75 \pm 9.96$ & $34.28 \pm 8.03$ & $33.14 \pm 6.22$ & $0.188(0.914)$ \\
\hline UACR (mg/dL/mg/dL) & $0.070[0.000-2.134]$ & $0.021[0.000-0.665]$ & $0.029[0.004-0.665]$ & $0.508[0.003-2.134]$ & $<0.001(0.001)$ \\
\hline $\mathrm{CRP}(\mathrm{mg} / \mathrm{dL}) *$ & $0.19[0.02-12.80]$ & $0.21[0.02-12.80]$ & $0.21[0.02-2.94]$ & $0.14[0.02-1.50]$ & $0.140(0.255)$ \\
\hline Renal length (mm) & $110.2 \pm 10.5$ & $110.5 \pm 10.5$ & $110.8 \pm 6.0$ & $109.5 \pm 10.8$ & $0.757(0.693)$ \\
\hline Cortical thickness (mm) & $14.3 \pm 2.9$ & $14.4 \pm 2.9$ & $16.0 \pm 2.5$ & $14.1 \pm 3.2$ & $0.689(0.069)$ \\
\hline
\end{tabular}

BMI body mass index; CKD-EPI—based on cystatin (Cys) and creatinine (Cr) chronic kidney disease epidemiology formula; GN-CKD—CKD due to glomerulonephritis; HT-CKD—hypertensive nephropathy; HTm-HT-CKD matched; * median [range] 
Table 2 Comparison of antihypertensive treatment between investigated groups

\begin{tabular}{llll}
\hline Kind of medication & HT-CKD $(n=38)(\%)$ & GN-CKD $(n=18)(\%)$ & $p$ value (HT/GN) \\
\hline ACE-I & 45.45 & 26.67 & 0.217 \\
ARB & 24.24 & 40.00 & 0.266 \\
BB & 73.53 & 46.67 & 0.069 \\
CCB & 45.45 & 40.00 & 0.724 \\
Diuretics & 78.79 & 66.67 & 0.369 \\
A1B & 24.24 & 13.33 & 0.388 \\
CN & 6.06 & 13.33 & 0.398 \\
\hline
\end{tabular}

$A C E-I$ angiotensin-converting enzyme inhibitor, $A R B$ angiotensin receptor blocker, $A 1 B$ a 1 adrenergic receptor blocker (doxazosin), $B B$ b-blocker, $C C B$ calcium channel blocker, $C N$ centrally acting agent (clonidine, $\alpha$-methyldopa), $G N$-CKD CKD due to glomerulonephritis, $H T$ - $C K D$ hypertensive nephropathy
Patients were not hypertensive according to ambulatory blood pressure measurements, and groups did not differ in 24-h blood pressure parameters, but LA, CI, E/E', and cIMT were higher in the HT-CKD group (Table 3).

Analysis of US Doppler perfusion parameters revealed significantly lower values of proximal RCP, total and especially proximal RCAA in the hypertension-related nephropathy group (Table 4; Fig. 1a, b). Values of distal RCP and distal RCAA did not differ between groups. Neither total nor proximal RCP and RCAA values were significantly correlated with their ROIs, renal lengths, and cortical thicknesses. Moreover, the perfusion and arterial area parameters were also not related to BMI.

To eliminate the difference in age and sample size, the oldest consecutive patients from HT-CKD group were excluded, and then remaining HT-CKD patients (HT-CKD matched group) were compared with GN-CKD patients. The groups did not differ in age, renal function, and cardiac properties, but total and proximal RCAA were significantly lower in HT-CKD matched group (Table 1, 3, 4).

In the whole population proximal RCP was significantly associated $(p<0.05)$ with age, CKD-EPI ${ }_{\text {Cys-Cr }}$, NT-proBNP, Troponin-I, E/A, E/E', LVEF, RI, and cIMT (Table 5), but in multivariable regression analysis adjusted to age (model without RI) only LVEF and cIMT were independently associated with proximal $\mathrm{RCP}\left(R^{2}=0.39 ; p<0.001\right)$.

ROC analysis showed that hypertensive kidney damage was identified with a sensitivity of $67 \%$ and a specificity of $68 \%$ with a threshold for $\mathrm{RCP} \leq 0.21 \mathrm{~mL} / \mathrm{s}$ (accuracy 0.679; AUC 0.715, $p=0.002$ ). Analysis performed for proximal RCAA showed significant correlations with age, etiology of kidney damage, NT-proBNP, $\mathrm{E} / \mathrm{A}, \mathrm{E} / \mathrm{E}^{\prime}$, RI, and cIMT. Although, in multivariable regression analysis adjusted to age only cIMT was independently associated with proximal RCAA $\left(R^{2}=0.25\right.$, $p<0.001)$, statistical power of pRCAA and etiology of

Table 3 Cardiovascular characteristics of investigated groups

\begin{tabular}{llllll}
\hline Data & Total $(n=56)$ & HT-CKD $(n=38)$ & $\begin{array}{l}\text { HT-CKD matched } \\
(n=18)\end{array}$ & GN-CKD $(n=18)$ & $\begin{array}{l}p \text { value HT/GN }(\mathrm{HTm} / \\
\text { GN })\end{array}$ \\
\hline Troponin-I $(\mathrm{ng} / \mathrm{mL})^{*}$ & $0.021[0.002-0.390]$ & $0.023[0.006-0.390]$ & $0.016[0.006-0.390]$ & $0.018[0.002-0.360]$ & $0.435(0.792)$ \\
NT-proBNP $(\mathrm{pg} / \mathrm{mL})^{*}$ & $91.55[10.60-$ & $\begin{array}{l}104.15[12.2- \\
27,785.0]\end{array}$ & $42.27[12.2-27,785.0]$ & $78.85[10.6-392.8]$ & $0.229(0.486)$ \\
& $27,785.0]$ & $0.889 \pm 0.197$ & $0.787 \pm 0.199$ & $0.712 \pm 0.177$ & $0.004(0.317)$ \\
cIMT $(\mathrm{mm})$ & $0.837 \pm 0.206$ & $3.97 \pm 0.50$ & $4.01 \pm 0.50$ & $3.67 \pm 0.51$ & $0.044(0.056)$ \\
LA $(\mathrm{cm})$ & $3.88 \pm 0.52$ & $104.82 \pm 35.54$ & $112.64 \pm 45.38$ & $100.83 \pm 25.99$ & $0.952(0.728)$ \\
LVMI $\left(\mathrm{g} / \mathrm{m}^{2}\right)$ & $103.56 \pm 32.64$ & $61.46 \pm 8.79$ & $61.59 \pm 6.76$ & $59.38 \pm 10.45$ & $0.450(0.530)$ \\
LVEF $(\%)$ & $60.79 \pm 9.31$ & $4.40 \pm 1.20$ & $3.88 \pm 1.21$ & $3.57 \pm 1.08$ & $0.017(0.575)$ \\
CI $\left(\mathrm{L} / \mathrm{min} / \mathrm{m}^{2}\right)$ & $4.13 \pm 1.21$ & $1.00 \pm 0.38$ & $1.14 \pm 0.44$ & $1.18 \pm 0.57$ & $0.240(0.945)$ \\
E/A & $1.06 \pm 0.45$ & $10.38 \pm 2.55$ & $10.44 \pm 3.11$ & $9.09 \pm 3.27$ & $0.020(0.089)$ \\
E/E & $10.00 \pm 2.80$ & $125.59 \pm 17.20$ & $127.88 \pm 16.48$ & $127.33 \pm 11.82$ & $0.525(0.597)$ \\
SBP $(\mathrm{mmHg})$ & $126.14 \pm 15.61$ & $75.59 \pm 12.56$ & $79.82 \pm 12.54$ & $78.94 \pm 8.36$ & $0.063(0.498)$ \\
DBP $(\mathrm{mmHg})$ & $76.65 \pm 11.43$ & & &
\end{tabular}

$C I$ cardiac index, E/A ratio of transmitral early (E) to late (A) ventricular filling velocities, $c I M T$ carotid intima-media thickness, $E / E^{\prime}$ ratio of transmitral early filling velocity to tissue Doppler early diastolic mitral annular velocity, $G N-C K D$ CKD due to glomerulonephritis, $H T$ - $C K D$ hypertension-related nephropathy, $H T m$ HT-CKD matched, $L A$ left atrium diameter, $L V E F$ left ventricular ejection fraction, $L V M I$ left ventricular mass index, SBP, DBP systolic, diastolic blood pressure; * median [range] 
Table 4 Differences in Doppler parameters of renal arteries in investigated groups

\begin{tabular}{llllll}
\hline Data & All $(n=56)$ & HT-CKD $(n=38)$ & HT-CKD matched $(n=18)$ & GN-CKD $(n=18)$ & $p$ value HT/GN $(\mathrm{HTm} / \mathrm{GN})$ \\
\hline RI (ratio) & $0.680 \pm 0.073$ & $0.689 \pm 0.078$ & $0.656 \pm 0.074$ & $0.659 \pm 0.056$ & $0.159(0.632)$ \\
tRCP $(\mathrm{mL} / \mathrm{s})$ & $0.307 \pm 0.242$ & $0.289 \pm 0.264$ & $0.240 \pm 0.145$ & $0.346 \pm 0.183$ & $0.052(0.411)$ \\
pRCP $(\mathrm{mL} / \mathrm{s})$ & $0.262 \pm 0.201$ & $0.243 \pm 0.216$ & $0.407 \pm 0.245$ & $0.305 \pm 0.163$ & $0.029(0.282)$ \\
tRCAA $\left(\mathrm{cm}^{2}\right)$ & $0.191 \pm 0.119$ & $0.178 \pm 0.131$ & $0.155 \pm 0.079$ & $0.220 \pm 0.085$ & $0.028(0.099)$ \\
pRCAA $\left(\mathrm{cm}^{2}\right)$ & $0.151 \pm 0.090$ & $0.138 \pm 0.095$ & $0.123 \pm 0.062$ & $0.181 \pm 0.068$ & $0.009(0.012)$ \\
pAAI (ratio) & $0.244 \pm 0.120$ & $0.216 \pm 0.101$ & $0.223 \pm 0.103$ & $0.302 \pm 0.137$ & $0.011(0.071)$ \\
\hline
\end{tabular}

$G N$-CKD CKD due to glomerulonephritis, $H T$-CKD hypertension-related nephropathy, HTm HT-CKD matched, $R I$ renal resistance index, $t R C P$, $p R C P$ total, proximal renal cortical perfusion, $t R C A A, p R C A A$ total, proximal renal cortical arterial area, $p A A I$ proximal arterial area index; * median [range]
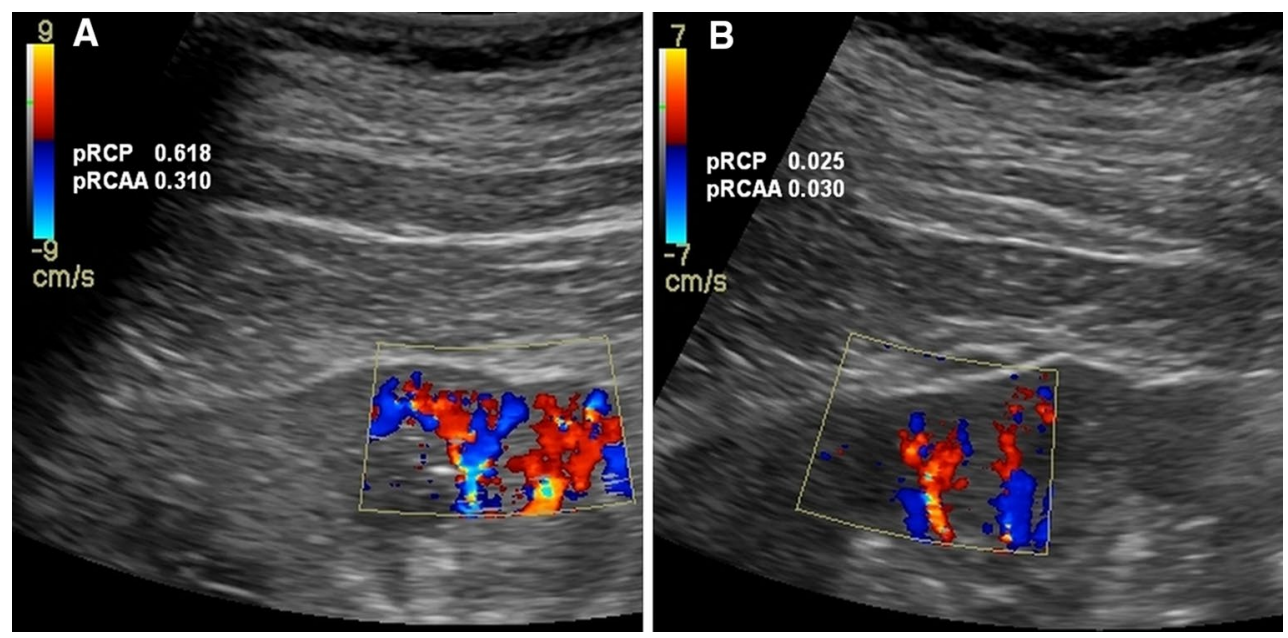

Fig. 1 Color Doppler imaging of renal cortical perfusion. Right kidney cortical perfusion imaging: a 61-year-old man with GN-CKD; b 60-year-old man with HT-CKD

Table 5 Significant correlations between perfusion parameters and other variables $(p<0.05)$

\begin{tabular}{lllllllllll}
\hline Data & CKD etiology & Age & CKD-EPI & Troponin-I & NT-proBNP & LVEF & E/A & E/E' & RI & CIMT \\
\hline pRCP & ns & -0.36 & 0.487 & -0.28 & -0.50 & 0.27 & 0.30 & -0.47 & -0.52 & -0.42 \\
pRCAA & 0.41 & -0.35 & ns & ns & -0.32 & ns & 0.27 & -0.30 & -0.36 & -0.37 \\
\hline
\end{tabular}

cIMT carotid intima-media thickness, CKD-EPI based on cystatin (Cys) and creatinine (Cr) chronic kidney disease epidemiology formula, E/A ratio of transmitral early (E) to late (A) ventricular filling velocities, $E / E^{\prime}$ ratio of transmitral early filling velocity to tissue Doppler early diastolic mitral annular velocity, $L V E F$ left ventricular ejection fraction, $R I$ renal resistance index, $n s$ not significant

kidney damage association was high $(0.90$, for the probability of type I error $=0.05$ ). Finally, although ROIs did not differ between groups, arterial vascularity (AAI) was significantly lower in HT-CKD compared to GN-CKD patients (Table 4).

Although comparison of proximal RCAA (AUC 0.753, $p<0.001)$ and proximal RCP in ROC analysis did not favor any of these parameters $(p=0.422)$, a threshold of $\leq 0.149 \mathrm{~cm}^{2}$ in proximal renal cortex arterial area
(RCAA) identified hypertension-related kidney damage with sensitivity of $71 \%$ and specificity of $78 \%$ (Fig. 2).

\section{Discussion}

This study shows for the first time that characteristics of US Doppler regional renal cortex perfusion could be helpful in distinguishing between hypertensive and 


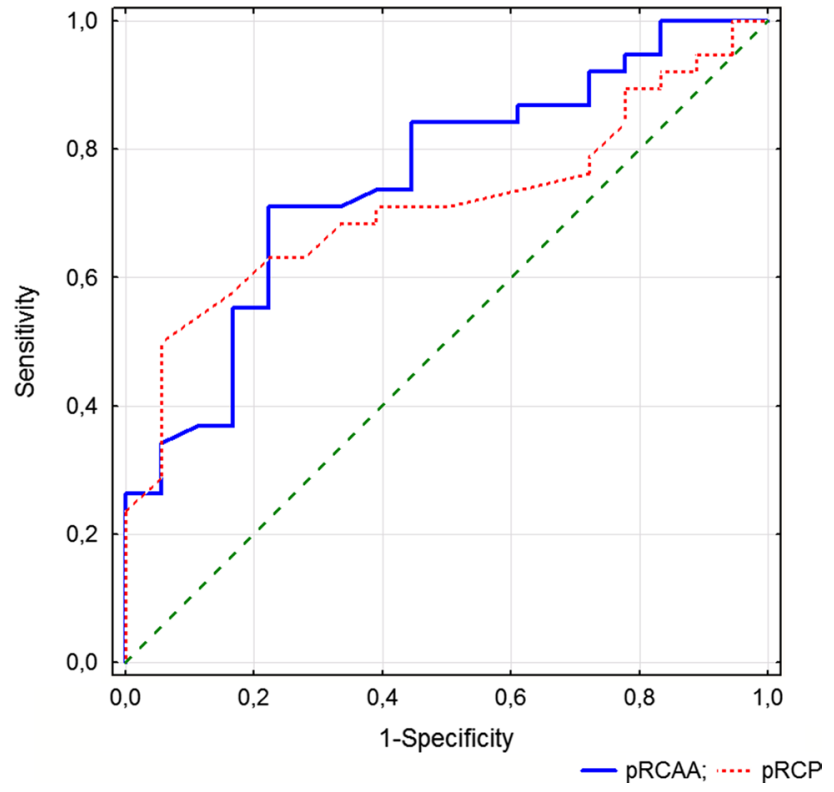

Fig. 2 Receiver operating characteristics (ROC) of total proximal renal cortical perfusion (tRCAA, full line, threshold $\leq 0.149 \mathrm{~cm}^{2}$ ) and proximal renal cortical perfusion ( $\mathrm{pRCP}$, dotted line, threshold $\leq 0.21 \mathrm{~mL} / \mathrm{s}$ ) to identify hypertension-related kidney damage

glomerulonephritis-related kidney damage. In ultrasound examinations we found a significant reduction in arterial vascularity in renal proximal cortex in patients with hypertensive kidney damage.

In general it is not difficult to establish the primary diagnosis in advanced stages of cardio-renal disorders [1]. Advanced cardio-renal disturbances are accompanied and preceded by important cardiovascular changes such as hypertension, coronary artery disease, or heart failure. Disorders primarily caused by renal disease are characterized by a decrease in urine specific gravity, changes in urinary sediment (erythrocyturia, leukocyturia), and proteinuria usually exceeding $1.0 \mathrm{~g} / 24 \mathrm{~h}$ so that the overall kidney damage exceeds that of the heart. Early diagnosis of the primary cause is the key to start an appropriate treatment and to stop or slow the progress of CKD [20]. However, in the initial stages of the disease, when both the cardiovascular and renal function parameters are within normal limits or only slightly affected, and data from the interview are inconclusive, it is difficult or impossible to identify the underlying cause. Proper identification of the initial etiology of cardio-renal disorders remains an ongoing challenge and is subject of many studies [21, 22]. The correct diagnosis is difficult because of the diversity of causes determining the variants of cardiac (right-/left-sided heart failure, with normal/decreased cardiac output, hypo-/hypertension) or renal dysfunctions (e.g., acute/chronic failure, with/without nephrotic syndrome, glomerular/tubulointerstitial/ischemic damage). Current research and diagnosis of cardio-renal syndrome focuses on identifying appropriate biomarkers such as creatinine, cystatin $\mathrm{C}$, brain natriuretic peptide (BNP), neutrophil gelatinase-associated lipocalin (NGAL), and kidney injury molecule-1 that would allow for an adequate recognition of the primary damage [22, 23]. Recently, Zhou et al. [24] showed an independent correlation of the urea-to-creatinine ratio and left ventricular diastolic dysfunction in 1166 community residents. In a different study, Testani et al. [21] proposed a combined assessment of BNP and blood urea nitrogen-to-creatinine ratio to predict the outcome in patients with decompensated heart failure and renal failure.

Due to the pathophysiology of cardio-renal syndrome and a significant contribution of both organ ischemia and an excess of extracellular volume, the assessment of renal perfusion appears to be a promising direction of research [1]. For example, Ciccone et al. [25] observed the usefulness of US Doppler assessment of renal perfusion in predicting the course of heart failure in 250 patients with heart failure. Schnell and Darmon [26] proposed to assess renal perfusion at the bedside using color Doppler and contrast-enhanced sonography to evaluate RI. We previously reported that RI only slightly correlated with renal perfusion [27]. However, Scholbach et al. [28] proposed an alternative method to quantify renal perfusion which has been termed dynamic tissue perfusion measurement (DTPM). Using this method we previously showed a significant independent association of perfusion intensity $(\mathrm{cm} / \mathrm{s})$ in the outer renal cortex with cardiac output, while the only factor independently associated with perfusion intensity in the proximal cortex turned out to be eGFR [5]. Furthermore, we showed that the renal perfusion index (RPI) succeeded in identifying the etiology of chronic cardio-renal syndrome with a sensitivity of $41.7 \%$ and a specificity of 83.3\% (AUC 0.597) [6].

In the study reported here the groups with different etiologies were comparable in terms of renal function, LVMI, as well as arterial blood pressures. Higher cardiac index in the hypertensive CKD group, despite the lack of differences in LVMI and EF, may have been related to adaptive changes [29]. Another explanation for different CI could be related to myocardial fibrosis as suggested by Edwards et al., who examined 43 patients with primary nephropathies or nephropathies dependent on vasculitis, and who found a significantly greater degree of myocardial fibrosis and significantly lower global longitudinal strain compared with 43 patients with hypertension but without renal failure [30]. This interpretation, however, can be disputed in the present study because of differences in renal function.

In the present study, we showed that arterial alterations expressed as cIMT and cardiac systolic function (LVEF) both had an independent influence on perfusion of renal proximal cortex. An estimated renal function was 
significantly correlated with this proximal RCP, and as it was previously reported, it was the only independent factor associated with RCP we could find [5]. Recently, Breidthardt et al. [31] published renal perfusion data measured by magnetic resonance imaging in heart failure patients in the presence or absence of cardio-renal syndrome. The authors also found a significant independent correlation of renal perfusion with eGFR confirming the adequacy of DTPM and the plausibility of our observations.

It is evident that organ perfusion should depend on cardiac function and vascular changes. Thickening of cIMT is an established marker of hypertensive target organ damage. Long-term hypertension results in rarefaction of microcirculatory vessels throughout the body, in the myocardium and in renal parenchyma [32]. Comparing the area of arteries we found a significant reduction in HT-CKD compared to GN-CKD patients, mainly in the proximal cortex. The threshold of $0.149 \mathrm{~cm}^{2}$ in proximal renal cortex arterial area was a reliable predictor differentiating the etiology of cardio-renal disturbances (sensitivity of $71 \%$, specificity of $78 \%$, AUC 0.753). This observation is new. Previous studies did not analyze the diagnostic potential of arterial area in the proximal renal cortex, obtained by DTPM to distinguish between cardio-renal and reno-cardiac disorders.

In our work, arterial density in the proximal cortex was evaluated as AAI. The significant reduction in arterial vascularity and, at the same time, renal perfusion in HT-CKD patients is probably due to continued pressure-related strain over extended periods of time as documented by higher cIMT, LA, and E/E' levels in the HT-CKD group [2, 33]. According to current knowledge, the direct arterial damage due to the increased pressure load can be associated with more severe inflammation in the renal cortex [34]. This can explain the differences in perfusion and vessel density in HT-CKD patients, especially because of reduced strain in patients with chronic, stable, and low-activity glomerulonephritis included in this study. Such a possibility is supported by studies of Scholbach et al. [35], who demonstrated a significant reduction in renal cortex perfusion assessed by DTPM with increasing peritubular inflammation.

It is clear that lower and deeply located ROI can easily affect the results of the ultrasound examination. However, in the present study, the assessed perfusion parameters were not significantly correlated with renal length, cortical thickness or ROI size, and BMI, which promotes the protocol and the method that we used. In the study by Tarnoki et al. [36], renal width appeared to be a better marker of the organ disease than renal length, which was rather connected with a genetic predisposition. In our work, the groups did not differ in renal length, but the cortical thickness tended to be even higher in the age-matched HT-CKD patients, which was in opposition to the lower arterial area in this group. However, this observation can be related to a direct arterial damage in the HT-CKD group and an expected more advanced renal interstitial fibrosis, and thus to the cortical diminishing as a consequence of chronic glomerulonephritis in GN-CKD patients. Elevated arterial stiffness, higher peak wave velocity (PWV), and cIMT are well-known markers of hypertensive target organ damage. In comparison with the GN-CKD group, in our study, cIMT was higher in the HT-CKD patients, which suggests more advanced arterial alterations in this group. Unfortunately, this difference appeared to be insignificant between the GN-CKD and the age-matched HT-CKD patients, which, however, can be related to a lower sensitivity of cIMT in the detection of arterial alterations in CKD patients [37]. In these conditions, a measurement of PWV or arterial wall stiffness would be a more appropriate examination showing arterial condition.

Despite promising results our work has several limitations. We have not assessed intra- or interobserver variability of DTPM method, because we used the best quality DICOM movie file for perfusion parameters calculation. Moreover, there are no such data in the available literature. In the only one recently published study by Stoperka et al. [38], the authors suggested limited accuracy of DTPM in diabetic patients. However, in this work, different color gain levels were used to achieve better-quality images, which is strictly restricted to this method, and keeping constant gain has a fundamental importance for the correctness of calculated results [14]. Our study involved only men so that the results cannot be extended to the females. Moreover, incoherent interviews concerning the origin and the precise duration of the disease, as well as the lack of histopathological confirmation could result in inappropriate determination of the causes of the observed differences. Patients with other causes of kidney diseases such as primary tubulointerstitial kidney injury, diabetes, amyloidosis, acute and advanced heart failure were excluded from the study to obtain homogeneous groups. This exclusion limits the usefulness of the test. Another limitation is an exclusion of renal artery stenosis based only on anamnesis and ultrasound Doppler examinations. On the other hand, the included patients did not have clinical indications for computed tomography or magnetic resonance diagnostics. Therefore, and in spite of the promising results of this study, the possibility of using a noninvasive assessment to diagnose the initial cause of cardio-renal disturbances needs to be confirmed in further studies.

\section{Conclusions}

Arterial vascularity and perfusion of renal proximal cortex could be helpful to diagnose the initial cause of cardiorenal disturbances in hypertensive males in early CKD 
stages. The utility of these measures to diagnose the primary cause of cardio-renal dysfunction in women requires further studies.

Funding This study was funded by Ministry of Science and Higher Education (Grant No. 331).

\section{Compliance with ethical standards}

Conflict of interest A. L, G. K, R. R, P. B, P. D, K. S, D. S, and S. N declare that they have no conflict of interest.

Ethical approval All procedures performed in studies involving human participants were in accordance with the ethical standards of the institutional and/or national research committee and with the 1964 Declaration of Helsinki and its later amendments or comparable ethical standards.

Informed consent Informed consent was obtained from all individual participants included in the study.

Open Access This article is distributed under the terms of the Creative Commons Attribution 4.0 International License (http://creativecommons.org/licenses/by/4.0/), which permits unrestricted use, distribution, and reproduction in any medium, provided you give appropriate credit to the original author(s) and the source, provide a link to the Creative Commons license, and indicate if changes were made.

\section{References}

1. McCullough PA, Kellum JA, Haase M, Müller C, Damman K, Murray PT et al (2013) Pathophysiology of the cardiorenal syndromes: executive summary from the eleventh consensus conference of the Acute Dialysis Quality Initiative (ADQI). Contrib Nephrol 182:82-98

2. Tomiyama H, Yamashina A (2015) Vascular dysfunction: a key player in chronic cardio-renal syndrome. Intern Med 54:1465-1472

3. Mancia G, Fagard R, Narkiewicz K, Redon J, Zanchetti A, Böhm $M$ et al (2014) 2013 ESH/ESC practice guidelines for the management of arterial hypertension. Blood Press 23:3-16

4. Humar R, Zimmerli L, Battegay E (2009) Angiogenesis and hypertension: an update. J Hum Hypertens 23:773-782

5. Lubas A, Ryczek R, Kade G, Smoszna J, Niemczyk S (2013) Impact of cardiovascular organ damage on cortical renal perfusion in patients with chronic renal failure. Biomed Res Int. doi: $10.1155 / 2013 / 137868$

6. Lubas A, Ryczek R, Kade G, Niemczyk S (2015) Renal perfusion index reflects cardiac systolic function in chronic cardiorenal syndrome. Med Sci Monit 21:1089-1096

7. Dyrla P, Lubas A, Gil J, Niemczyk S (2016) Doppler tissue perfusion parameters in recognizing pancreatic malignant tumors. $\mathbf{J}$ Gastroenterol Hepatol. doi:10.1111/jgh.13193

8. Woźniak MM, Scholbach TM, Scholbach J, Pawelec A, Nachulewicz P, Wieczorek AP et al (2016) Color Doppler dynamic tissue perfusion measurement: a novel tool in the assessment of renal parenchymal perfusion in children with vesicoureteral reflux. Arch Med Sci. doi:10.5114/aoms.2015.51698

9. Lubas A, Kade G (2010) Imaging methods in quantitative assessment of renal perfusion. Lek Wojsk 88:403-407
10. Scholbach TM, Vogel C, Bergner N (2014) Color Doppler sonographic dynamic tissue perfusion measurement demonstrates significantly reduced cortical perfusion in children with diabetes mellitus type 1 without microalbuminuria and apparently healthy kidneys. Ultraschall Med 35:445-450. doi:10.105 5/s-0034-1365909

11. Scholbach T, Wang HK, Yang AH, Loong CC, Wu TH (2013) Correlation of histopathologic and dynamic tissue perfusion measurement findings in transplanted kidneys. BMC Nephrol. doi:10.1186/1471-2369-14-143

12. National Kidney Foundation (2002) K/DOQI clinical practice guidelines for chronic kidney disease: evaluation, classification, and stratification. Am J Kidney Dis 39(2 Suppl 1):S1-266

13. Levey AS, Stevens LA, Schmid CH, Zhang YL, Castro AF, Feldman HI et al (2009) A new equation to estimate glomerular filtration rate. Ann Intern Med 150:604-612

14. Scholbach T, Dimos I, Scholbach J (2004) A new method of color Doppler perfusion measurement via dynamic sonographic signal quantification in renal parenchyma. Nephron Physiol 96:99-104

15. Sahn DJ, DeMaria A, Kisslo J, Weyman A (1978) Recommendations regarding quantitation in M-mode echocardiography: results of a survey of echocardiographic measurements. Circulation 58:1072-1083

16. Devereux RB, Alonso DR, Lutas EM, Gottlieb GJ, Campo E, Sachs I et al (1986) Echocardiographic assessment of left ventricular hypertrophy: comparison to necropsy findings. Am J Cardiol 57:450-458

17. Mosteller RD (1987) Simplified calculation of body surface area. N Engl J Med 317:1098

18. Lang RM, Bierig M, Devereux RB, Flachskampf FA, Foster E, Pellikka PA, Picard MH et al (2006) Recommendations for chamber quantification. Eur J Echocardiogr 7:79-108

19. Krumme B, Blum U, Schwertfeger E, Flügel P, Höllstin F, Schollmeyer P, Rump LC (1996) Diagnosis of renovascular disease by intra- and extrarenal Doppler scanning. Kidney Int 50:1288-1292

20. Clementi A, Virzì GM, Brocca A, de Cal M, Vescovo G, Granata A, Ronco C (2013) Cardiorenal syndrome type 4: management. Blood Purif 36:200-209

21. Testani JM, Damman K, Brisco MA, Chen S, Laur O, Kula AJ et al (2014) A combined-biomarker approach to clinical phenotyping renal dysfunction in heart failure. J Card Fail 20:912-919

22. Cruz DN, Schmidt-Ott KM, Vescovo G, House AA, Kellum JA, Ronco C, McCullough PA (2013) Pathophysiology of cardiorenal syndrome type 2 in stable chronic heart failure: workgroup statements from the eleventh consensus conference of the Acute Dialysis Quality Initiative (ADQI). Contrib Nephrol 182:117-136

23. Brisco MA, Testani JM (2014) Novel renal biomarkers to assess cardiorenal syndrome. Curr Heart Fail Rep 11:485-499

24. Zhou J, Cui X, Jin X, Zhou J, Zhang H, Tang B et al (2014) Association of renal biochemical parameters with left ventricular diastolic dysfunction in a community-based elderly population in China: a cross-sectional study. PLoS ONE. doi:10.1371/journal. pone. 0088638

25. Ciccone MM, Iacoviello M, Gesualdo L, Puzzovivo A, Antoncecchi V, Doronzo A et al (2014) The renal arterial resistance index: a marker of renal function with an independent and incremental role in predicting heart failure progression. Eur J Heart Fail 16:210-216

26. Schnell D, Darmon M (2015) Bedside Doppler ultrasound for the assessment of renal perfusion in the ICU: advantages and limitations of the available techniques. Crit Ultrasound J. doi:10.1186/ s13089-015-0024-6 
27. Lubas A, Kade G, Niemczyk S (2013) Renal resistive index as a marker of vascular damage in cardiovascular diseases. Int Urol Nephrol 46:395-402

28. Scholbach T, Scholbach J (2009) Can we measure renal tissue perfusion by ultrasound? J Med Ultrasound 17:9-16

29. Sinkeler SJ, Damman K, van Veldhuisen DJ, Hillege H, Navis G (2012) A re-appraisal of volume status and renal function impairment in chronic heart failure: combined effects of pre-renal failure and venous congestion on renal function. Heart Fail Rev $17: 263-270$

30. Edwards NC, Moody WE, Yuan M, Hayer MK, Ferro CJ, Townend JN, Steeds RP (2015) Diffuse interstitial fibrosis and myocardial dysfunction in early chronic kidney disease. Am J Cardiol 115:1311-1317

31. Breidthardt T, Cox EF, Squire I, Odudu A, Omar NF, Eldehni MT et al (2015) The pathophysiology of the chronic cardiorenal syndrome: a magnetic resonance imaging study. Eur Radiol 25:1684-1691

32. Amann K, Breitbach M, Ritz E, Mall G (1998) Myocyte/capillary mismatch in the heart of uremic patients. J Am Soc Nephrol 9:1018-1022

33. Sharp AS, Tapp RJ, Thom SA, Francis DP, Hughes AD, Stanton $\mathrm{AV}$ et al (2010) Tissue Doppler E/E' ratio is a powerful predictor of primary cardiac events in a hypertensive population: an ASCOT substudy. Eur Heart J 31:747-752

34. Pauletto P, Rattazzi M (2006) Inflammation and hypertension: the search for a link. Nephrol Dial Transplant 21:850-853

35. Scholbach T, Wang HK, Yang AH, Loong CC, Wu TH (2013) Correlation of histopathologic and dynamic tissue perfusion measurement findings in transplanted kidneys. BMC Nephrol. doi:10.1186/1471-2369-14-143

36. Tarnoki DL, Tarnoki AD, Bata P, Littvay L, Garami Z, Berczi V, Karlinger K (2015) Different genetic impact in the development of renal length and width: a twin study. Intern Med J 45:63-67. doi:10.1111/imj.12631

37. Claridge M, Wilmink T, Ferring M, Dasgupta I (2015) Measurement of arterial stiffness in subjects with and without renal disease: Are changes in the vessel wall earlier and more sensitive markers of cardiovascular disease than intima media thickness and pulse pressure? Indian J Nephrol 25:21-26. doi:10.4103/0971-4065.138692

38. Stoperka F, Karger C, Beige J (2016) Limited Accuracy of colour Doppler ultrasound dynamic tissue perfusion measurement in diabetic adults. PLoS ONE. doi:10.1371/journal.pone.0168905 